Article DOI: https://doi.org/10.3201/eid2712.210491

\title{
Novel Filoviruses, Hantavirus, and Rhabdovirus in Freshwater Fish, Switzerland, 2017
}

\section{Appendix}

\section{Methods}

\section{Bioinformatics}

Reads were quality-trimmed using trimmomatic v. 0.36 (28), and host-derived sequences were removed by aligning reads to the European perch genome (UTU_Pfluv_1.1, Bioproject PRJNA450919) using STAR v. 2.6.0c (1). Non-aligned reads were assembled with SPAdes v. 3.12.0 (2). The resulting scaffolds were screened for homologies on the nucleotide and amino acid levels using BLASTn v. 2.7.1+(3) against viral nucleotide sequences in GenBank (https://www.ncbi.nlm.nih.gov/genbank/) and DIAMOND v. 0.9.18 (4) and against viral protein sequences in UniProt (https://www.uniprot.org/), respectively. (Databases were downloaded on 20 May 2020.)

\section{RT-PCR, RACE, Sanger sequencing}

To fill gaps between HTS scaffolds, we reverse-transcribed extracted RNA to cDNA with SuperScript III Reverse Transcriptase (Thermo Fisher Scientific, Waltham, MA, USA) and performed PCR assays with Q5 Hot Start High-Fidelity DNA Polymerase (New England Biolabs, Ipswhich, MA, USA) and scaffold-specific primers (Appendix Table 3) according to the manufacturers' instructions. We gel-purified amplicons using the NucleoSpin Gel \& PCR Cleanup Kit (Macherey-Nagel, Oensingen, Switzerland) and sequenced them using a 3730 DNA Analyzer (Thermo Fisher Scientific) with the BigDye Terminator v3.1 Cycle Sequencing Kit (Thermo Fisher Scientific), using standard protocols. We performed 3' and 5' RACE, as described previously on RNA extracted from pooled organs and CNS as well as cell culture supernatants (5). We purified RACE products and sequenced them as described above. Resultant data were analyzed with Geneious v 9.1.8 (Biomatters, Auckland, New Zealand). 


\section{In Situ hybridization (ISH)}

We conducted chromogenic ISH on all of the FFPE tissues used for histopathology. Staining was performed with the RNAscope system (Advanced Cell Diagnostics, Newark, CA, USA). Using the RNAscope 2.5 HD Assay-Brown according to the manufacturer's instructions. ISH probes were designed by the company for EGLV (catalog \#590061), BRPV (\#590031), FIWIV (\#590041), and OBLV (\#590051). We counterstained slides with Mayer's hemalum solution (Merck KGaA, Darmstadt, Germany) and mounted them with Aquatex (Merck KGaA). Sections of apparently healthy European perch from a different origin, which we examined for a normal health control, served as negative controls. ISH process controls consisted of brain tissue sections of animals with bovine astrovirus $\mathrm{CH} 13$ (BoAstV CH13) infection and a BoAstV CH13-specific RNAscope probe [\#406921] tested in parallel to each ISH experiment (6).

\section{References}

1. Dobin A, Davis CA, Schlesinger F, Drenkow J, Zaleski C, Jha S, et al. STAR: ultrafast universal RNAseq aligner. Bioinformatics. 2013;29:15-21. PubMed https://doi.org/10.1093/bioinformatics/bts635

2. Bankevich A, Nurk S, Antipov D, Gurevich AA, Dvorkin M, Kulikov AS, et al. SPAdes: a new genome assembly algorithm and its applications to single-cell sequencing. J Comput Biol. 2012;19:455-77. PubMed https://doi.org/10.1089/cmb.2012.0021

3. Camacho C, Coulouris G, Avagyan V, Ma N, Papadopoulos J, Bealer K, et al. BLAST+: architecture and applications. BMC Bioinformatics. 2009;10:421. PubMed https://doi.org/10.1186/1471$\underline{2105-10-421}$

4. Buchfink B, Xie C, Huson DH. Fast and sensitive protein alignment using DIAMOND. Nat Methods. 2015;12:59-60. PubMed https://doi.org/10.1038/nmeth.3176

5. Hierweger MM, Werder S, Seuberlich T. Parainfluenza virus 5 infection in neurological disease and encephalitis of cattle. Int J Mol Sci. 2020;21:498. PubMed https://doi.org/10.3390/ijms21020498

6. Küchler L, Rüfli I, Koch MC, Hierweger MM, Kauer RV, Boujon CL, et al. Astrovirus-associated polioencephalomyelitis in an alpaca. Viruses. 2020;13:50. PubMed https://doi.org/10.3390/v13010050 
Appendix Table 1. Results of the bioinformatics pipeline for virus discovery in samples from European perch*

\begin{tabular}{|c|c|c|c|c|c|c|c|}
\hline \multirow[b]{2}{*}{ Virus family } & \multicolumn{3}{|c|}{ Scaffold } & \multirow{2}{*}{$\begin{array}{c}\text { DIAMOND best protein hit } \\
\text { Accesssion number|protein|virus }\end{array}$} & \multicolumn{3}{|c|}{ Amino acid alignment } \\
\hline & ID & Length & kmer coverage & & Identity (\%) & Length [nt] & Query coverage (\%) \\
\hline \multirow{3}{*}{ Hantaviridae } & 10 & 6509 & 3010 & A0A2P1GNS4|Large protein|W. red spikefish virus & 34.4 & 2104 & 94.8 \\
\hline & 59 & 3784 & 11122 & A0A2P1GNS8|Glycoprotein|W. minipizza batfish virus & 25.4 & 836 & 63.1 \\
\hline & 550 & 2160 & 15043 & A0A2P1GNX7|Nucleoprotein|W. red spikefish virus & 30.3 & 330 & 42.4 \\
\hline \multirow[t]{16}{*}{ Rhabdoviridae } & 2058 & 1454 & 5.8 & Q8UYI1|Nucleocapsid protein|sea trout rhabdovirus & 42.3 & 267 & 54.8 \\
\hline & 4052 & 1146 & 3 & Q8UY99|Glycoprotein|lake trout rhabdovirus & 87.5 & 80 & 97.2 \\
\hline & 6439 & 960 & 5.7 & Q8V316|Phosphoprotein|sea trout rhabdovirus & 62.2 & 74 & 67.5 \\
\hline & 8194 & 873 & 1.9 & K7X7F6|Large protein|perch rhabdovirus & 95.2 & 84 & 89.4 \\
\hline & 10813 & 771 & 5.2 & Q8V315|Matrix protein|sea trout rhabdovirus & 94.8 & 213 & 82.9 \\
\hline & 10889 & 768 & 1.9 & A0A0A7|Large protein|eel virus European $X$ & 76.4 & 254 & 98.8 \\
\hline & 12097 & 731 & 2.4 & K7X7F6|Large protein|perch rhabdovirus & 87.2 & 243 & 99.7 \\
\hline & 37625 & 394 & 1 & Q8V317|Large protein|lake trout rhabdovirus & 93.2 & 426 & 87.9 \\
\hline & 42117 & 370 & 2 & K7X7F6|Large protein|perch rhabdovirus & 89 & 354 & 92.7 \\
\hline & 52025 & 331 & 1.4 & Q8V317|Large protein|lake trout rhabdovirus & 93.8 & 96 & 99.7 \\
\hline & 52641 & 329 & 1.1 & K7X7F6|Large protein|perch rhabdovirus & 84.4 & 122 & 98.9 \\
\hline & 68156 & 289 & 1.3 & Q8UY99|Glycoprotein|lake trout rhabdovirus $903 / 87$ & 77.6 & 303 & 94.7 \\
\hline & 71803 & 282 & 0.96 & Q8V313|Large protein|sea trout rhabdovirus & 66.7 & 84 & 98.8 \\
\hline & 89460 & 256 & 2.4 & K7X7F67|Large sprotein|perch rhabdovirus & 98.2 & 110 & 99.7 \\
\hline & 90246 & 255 & 3.8 & K7X7F6|Large protein|perch rhabdovirus & 82.1 & 84 & 98.4 \\
\hline & 97442 & 247 & 1.7 & Q8V313|Polymerase protein |sea trout rhabdovirus & 90.8 & 130 & 99 \\
\hline \multirow[t]{10}{*}{ Filoviridae } & 4 & 14593 & 23.5 & A0A2P1GMM1|Large protein|Huángjiāo virus & 50.3 & 843 & 17.6 \\
\hline & 5 & 13764 & 15.2 & A0A2P1GMM1|Large protein|Huángjiāo virus & 59.8 & 2145 & 46.5 \\
\hline & $14^{\dagger}$ & 10362 & 4.8 & A0A2P1GMM1|Large protein|Huángjiāo virus & 68 & 1471 & 42.5 \\
\hline & 1005 & 3259 & 3 & A0A2P1GMM1|Large protein|Huángjiāo virus & 47.1 & 1089 & 99.9 \\
\hline & 10054 & 3259 & 3 & A0A2P1GMM1|Large protein|Huángjiāo virus & 47.1 & 1089 & 99.9 \\
\hline & $5282 \dagger$ & 1945 & 4.1 & A0A2P1GMM1|Large protein|Huángjiāo virus & 41 & 648 & 99.6 \\
\hline & 7085 & 1729 & 2.2 & A0A2P1GMM5|Nucleoprotein|Huángjiāo virus & 38.8 & 389 & 66.8 \\
\hline & 11776 & 1389 & 1.8 & A0A2P1GMM1|Large protein|Huángjiāo virus & 41.6 & 334 & 71.7 \\
\hline & $34171 \dagger$ & 779 & 2.6 & A0A2P1GMM5|Nucleoprotein|Huángjiāo virus & 61.6 & 250 & 96.3 \\
\hline & 50926 & 573 & 3 & A0A2P1GMM1|Large protein|Huángjiāo virus & 58.4 & 190 & 99.5 \\
\hline \multirow[t]{3}{*}{ Other } & 1 & 10069 & 95 & A0A119QNF6|Capsid protein|marbled eel polyomavirus & 27.1 & 491 & 13.8 \\
\hline & 201 & 2764 & 6.8 & A0A1S7J028|LargeT|Rousettus aegyptiacus polyomavirus 1 & 28.6 & 255 & 26.6 \\
\hline & 44589 & 359 & 0.7 & A0A2P1GNG4|Polyprotein|Běihăi rabbitfish_calicivirus & 57.4 & 115 & 95.3 \\
\hline
\end{tabular}

†These scaffolds were linked by RT-PCR resulting in the sequence of a novel virus here named Kander virus (KNDV). 
Appendix Table 2. Comparison of conserved terminal sequences in genome segments of selected genera of the order Bunyavirales*

\begin{tabular}{|c|c|c|}
\hline Genus & 3' terminus & 5' terminus \\
\hline Orthohantavirus & AUCAUCAUCUG... & ...AUGAUGAU \\
\hline Orthobunyavirus & UCAUCACAUGA... & ...UCGUGUGAUGA \\
\hline Orthonairovirus & AGAGUUUCU... & ...AGAAACUCU \\
\hline Orthotospovirus & UCUCGUUAG... & ...CUAACGAGA \\
\hline Phlebovirus & UGUCGUUAG... & ...CUAACGAGA \\
\hline $\begin{array}{l}\text { Actinovirus } \\
\text { (Species: Perch actinovirus, Bunyavirales) }\end{array}$ & UCAUCAUU... & ...AAUGAUGA \\
\hline
\end{tabular}

(Species: Perch actinovirus, Bunyavirales)

${ }^{*}$ Adapted from Barr JN, Weber F, Schmaljohn CS. Bunyavirales: the viruses and their replication. In: Howley PM, Knipe DM, Whelan SPJ, editors. Fields virology. 7th ed. Philadelphia, Pennsylvania, USA: Wolters Kluwer/Lippincott Williams \& Wilkins; 2020. p. 706-49.

Appendix Table 3. Gene-specific primers for RT-PCRs, Sanger sequencing, and RACE

\begin{tabular}{|c|c|c|}
\hline Name & Sequence & Comment \\
\hline RT FiloV 3140F & TGTGAGCTCACCAACCGTAC & Confirmation \\
\hline RT_FiloV_3483R & GAGCCGTTTCTCCCAAGACA & Confirmation \\
\hline RT_HantaV_2950F & CCCGGAAGTCCAGAACCTTC & confirmation \\
\hline RT_HantaV_3269R & CGGTCAGGGAATCATCGGAG & confirmation \\
\hline RT_RhabdoV_1064F & AAATGCCATTGCCAACACCG & confirmation \\
\hline RT_RhabdoV_1370R & GTACGCTCCGACAGTGTCTT & confirmation \\
\hline RT_HantaV_L_493R & AAACTGAAGCTCGATGCCCA & RACE \\
\hline $\mathrm{RT}^{-}$HantaV L $\mathrm{L}^{-} 322 \mathrm{R}$ & CCAGCTGCCCAGGGAATATC & RACE \\
\hline RT_HantaV_L_5998F & CGTCAGGTCCTGGATCAAGG & RACE \\
\hline RT_HantaV_L_6190F & CTCCGCTGTGAACATGGTTG & RACE \\
\hline $\mathrm{RT}^{-} \mathrm{HantaV}^{-} \mathrm{M}^{-} 541 \mathrm{R}$ & TTCTGCCGCCTTTCAAAGC & RACE \\
\hline RT_HantaV_M_305R & CTCTTGGATCCTGGGTGTCG & RACE \\
\hline RT_HantaV_M_3273F & ACATAGGCACTGTCCTCAAGC & RACE \\
\hline $\mathrm{RT}^{-} \mathrm{HantaV}^{-} \mathrm{M}^{-} 3466 \mathrm{~F}$ & TTCCGACGAGACCTCCTTCT & RACE \\
\hline RT_HantaV_S_526R & CAGCCTGTGTTTCCGGAGTA & RACE \\
\hline RT_HantaV_S_281R & GCTGGATCTGAAGGCAGGAG & RACE \\
\hline RT_HantaV_S_1656F & CCCAAACAGGTCGGTCATCA & RACE \\
\hline RT_HantaV_S_1848F & CAAGGTGGTCTCCATGGGG & RACE \\
\hline RT_RhabdoV_1335F & ATCCAACATGCCCGCAAAGA & connection of contigs \\
\hline RT_RhabdoV_1684R & TCССАGСТССАСТААТСССТ & connection of contigs \\
\hline $\mathrm{RT}^{-}$RhabdoV_2391F & GAATGGAATGGATGCCAGCG & connection of contigs \\
\hline RT_RhabdoV_2863R & GCCACGACCATCGCATTTTT & connection of contigs \\
\hline RT_RhabdoV_3358F & GCAATGAGAAAGTGCTGAACCA & connection of contigs \\
\hline RT_RhabdoV_3750R & CCCATGCCGCAGTTTGATAC & connection of contigs \\
\hline $\mathrm{RT}^{-}$RhabdoV_3765F & GTAGAGGGGAAGTTGTGCGT & connection of contigs \\
\hline RT_RhabdoV_4214R & CTGCTGTCGAACGATTGCAC & connection of contigs \\
\hline RT_RhabdoV_5913F & GTTGGGCGTATGCAGACTCT & connection of contigs \\
\hline $\mathrm{RT}^{-}$RhabdoV $6225 \mathrm{R}$ & GCTCTGCGTTGACAAAGTCA & connection of contigs \\
\hline RT_RhabdoV_6256F & GATCTTTTCGGCATTGGGGC & connection of contigs \\
\hline RT_RhabdoV_6559R & TCGAAGATTTTGGTCAGAGGCA & connection of contigs \\
\hline $\mathrm{RT}^{-}$RhabdoV_6636F & CATGTAGAACGACGGCCCTT & connection of contigs \\
\hline RT_RhabdoV_7823R & TCCTCGAATTGCCGGATTGT & connection of contigs \\
\hline RT_RhabdoV_7793F & AGTAGAGTTTCACAATCCGGCA & connection of contigs \\
\hline $\mathrm{RT}^{-}$RhabdoV $8342 \mathrm{R}$ & CAGCGATATCAGGGATTCGGT & connection of contigs \\
\hline RT_RhabdoV_8780F & GCTGAGAGCCGCTATCACAT & connection of contigs \\
\hline RT_RhabdoV_9479R & GGACAACGCAGATGCCTTGA & connection of contigs \\
\hline RT_RhabdoV_10828F & GAGGCTCGACAGGGATCAAG & connection of contigs \\
\hline RT_RhabdoV_11124R & TСTTСТССССТTTGСAАСTGA & connection of contigs \\
\hline RT_Rhabdo_6952F & AGGAGTCAAGGGCAAGGAGA & connection of contigs \\
\hline RT_Rhabdo_7555R & GCTGACTGGATTGTCTCGTCA & connection of contigs \\
\hline $\mathrm{RT}^{-}$Rhabdo $6980 \mathrm{~F}$ & ACGAGCACATCAGTATTGCCA & connection of contigs \\
\hline RT_Rhabdo_575R & CTCGTTTTGGATCCCCACGA & RACE \\
\hline RT_Rhabdo_330R & CCAATTCGCTCGGCAATCTG & RACE \\
\hline $\mathrm{RT}^{-}$Rhabdo $11050 \mathrm{~F}$ & CGAATCTCTGCTTGCAGTGC & RACE \\
\hline RT_Rhabdo_11222F & AGGAAGATGGCCACCATTGG & RACE \\
\hline P_FiloV_13to3360_1435F & CACAGTGGTAAGGGCCATGA & connection of contigs \\
\hline $\mathrm{P}^{-}$FiloV 13 1303360-1884R & CCGGTGTCAACGGCTGTTAT & connection of contigs \\
\hline P_FiloV_13to5716_12038F & GTCTGCATAGGGAAGGTGGC & connection of contigs \\
\hline P_FiloV_13to5716_12590R & CTTGTCGCTCCTGAAGACGT & connection of contigs \\
\hline Perch_FiloV2_296R & TTGCAAGAATGAAGGCACACC & RACE \\
\hline Perch FiloV2-511R & AAACCTTGCGGCTTGATTGG & RACE \\
\hline Perch FiloV2 $14328 \mathrm{~F}$ & ACGCCTTGGTCAAATCCACA & RACE \\
\hline Perch_FiloV2_14118F & TTGCTCTTGTGGCAGCAGAA & RACE \\
\hline
\end{tabular}




\begin{tabular}{lcc}
\hline Name & Sequence & Comment \\
\hline Perch_FiloV1_260R & GTCGACTGAGTTGTCTCCCC & RACE \\
Perch_FiloV1_455R & GTATGGTAATGCCGTTGGTGC & RACE \\
Perch_FiloV1_13492F & TCCTAGGGAGTCTGCAGAGG & RACE \\
Perch_FiloV1_13275F & GACAGGTCCGGAGTGAAGTC & RACE \\
\hline
\end{tabular}
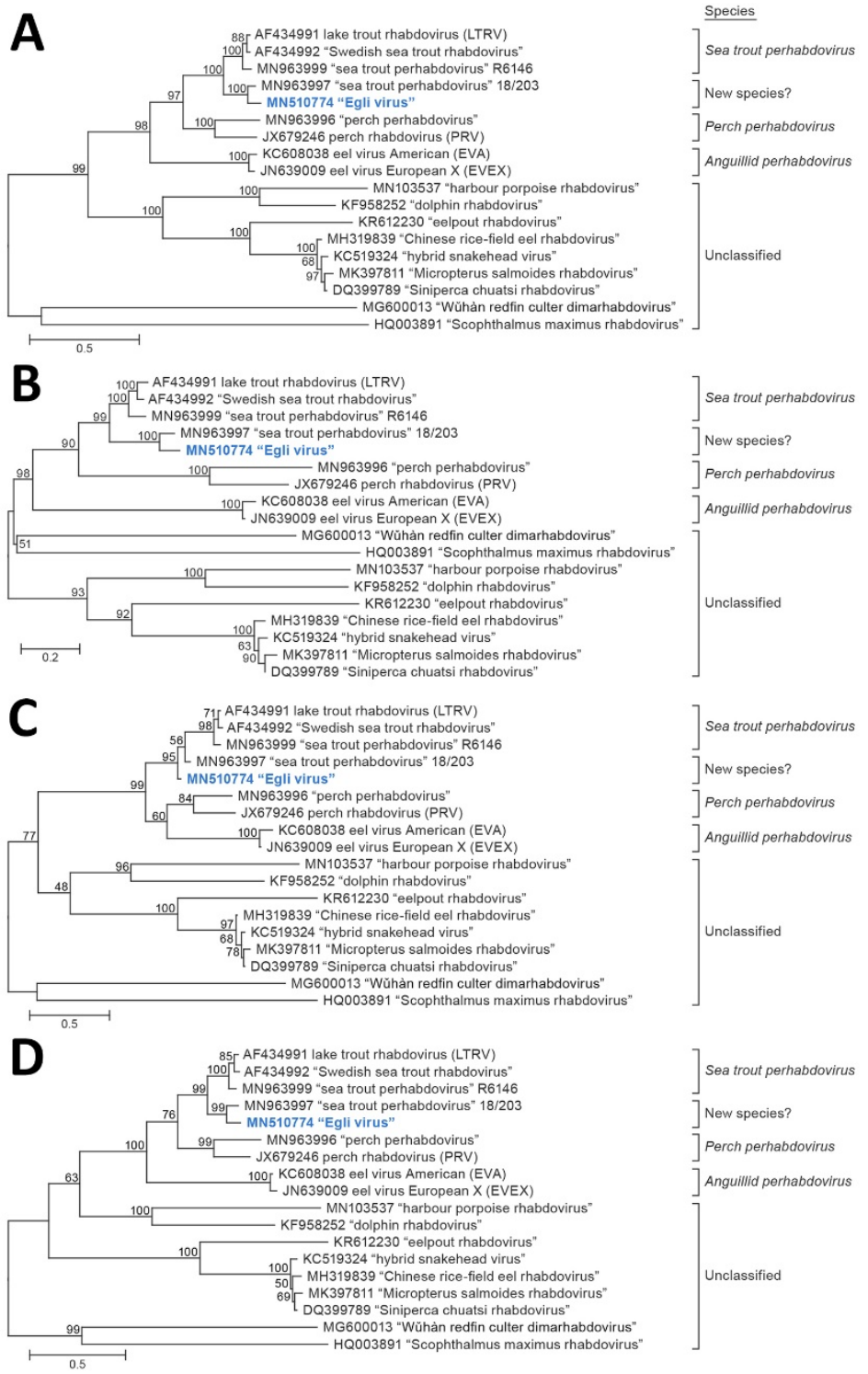

Appendix Figure 1. Maximum-likelihood phylogenetic trees of the nucleotide sequences of Egli virus (EGLV; bold blue) genes with those of viruses belonging to representative members of the genus Perhabdovirus. a) nucleoprotein gene $(N)$, b) phosphoprotein gene $(P)$ c) matrix protein gene $(M)$, and d) glycoprotein gene $(G)$. Numbers near nodes on the trees indicate bootstrap values. Branches are labeled by GenBank accession number, virus name, and virus name abbreviation in parenthesis. The names of unclassified, likely perhabdoviruses are placed in quotation marks and printed without name abbreviations. The scale (bottom left) indicates the number of substitutions per site, reflected by the branch lengths. 


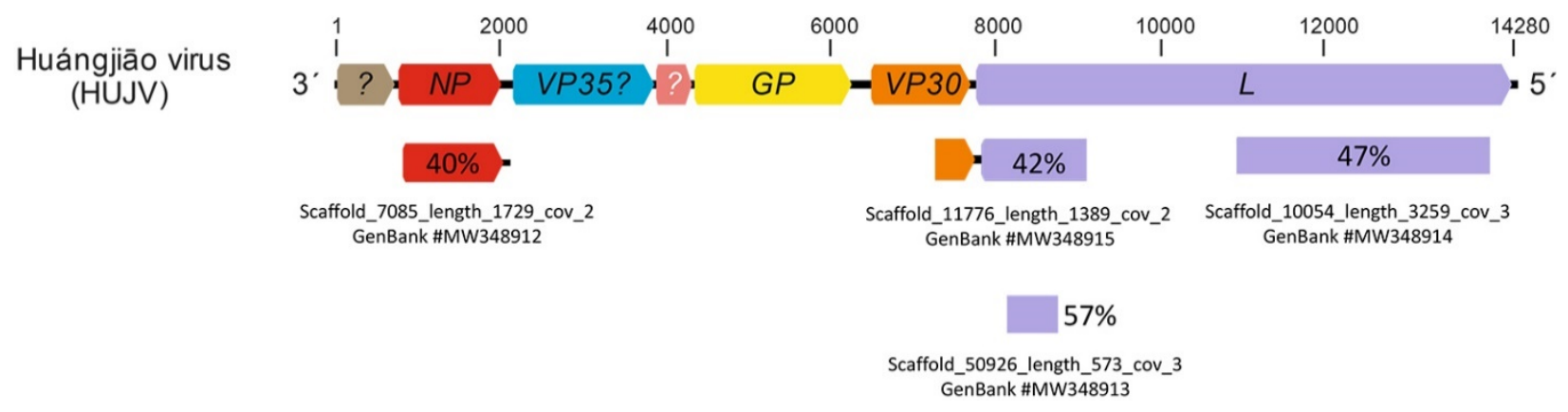

Appendix Figure 2. Mapping of additional scaffolds identified in European perch with hits to Huángjiāo virus (HUJV). The HUJV genome organization is shown schematically on the top and allocated scaffolds (scaffold IDs [shortened] and GenBank accession numbers) to the genomic regions of the encoded proteins with respective hits and their identity at the amino-acid sequence level. Open reading frames (ORFs) are indicated as colored arrows. ORFs encoding HUJV-like proteins (indicated as percentages) are depicted by the same color. NP, nucleoprotein gene; VP35, polymerase cofactor gene; GP, glycoprotein gene; VP30, transcriptional activator gene; $L$, large protein gene. Question marks indicate novel ORFs. 


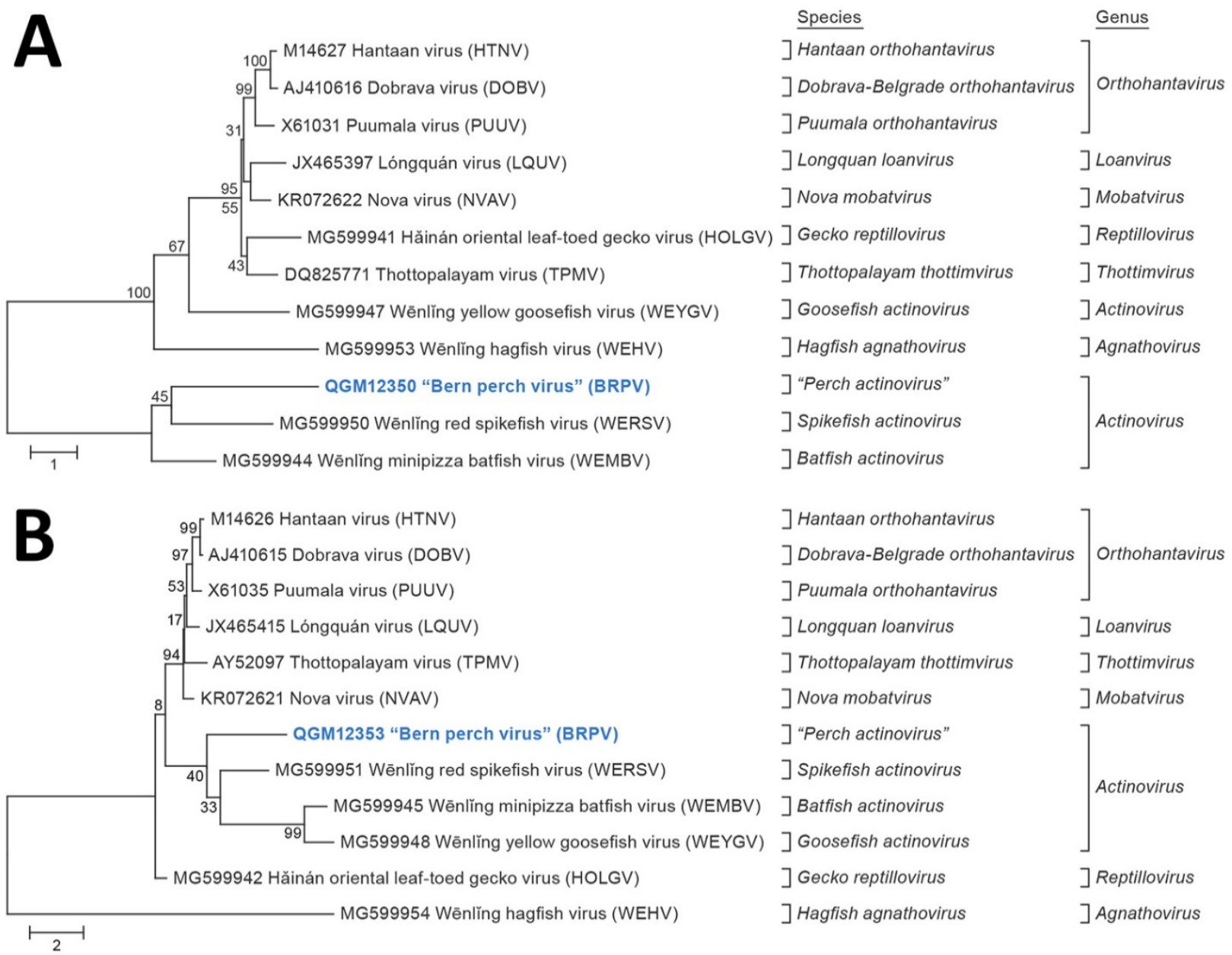

Appendix Figure 3. Maximum-likelihood phylogenetic trees of amino-acid sequences of Bern perch virus (BRPV; bold blue) structural proteins with those of viruses belonging to representative members of the family Hantaviridae. a) Glycoprotein precursor (GPC), encoded by the M segment, b) nucleocapsid protein, encoded by the $S$ segment. Numbers near nodes on the trees indicate bootstrap values. Branches are labeled by GenBank accession number, virus name, and virus name abbreviation in parenthesis. Unclassified, likely hantaviruses and officially proposed hantavirus species names are placed in quotation marks. The scale (bottom left) indicates the number of substitutions per site, reflected by the branch lengths. 

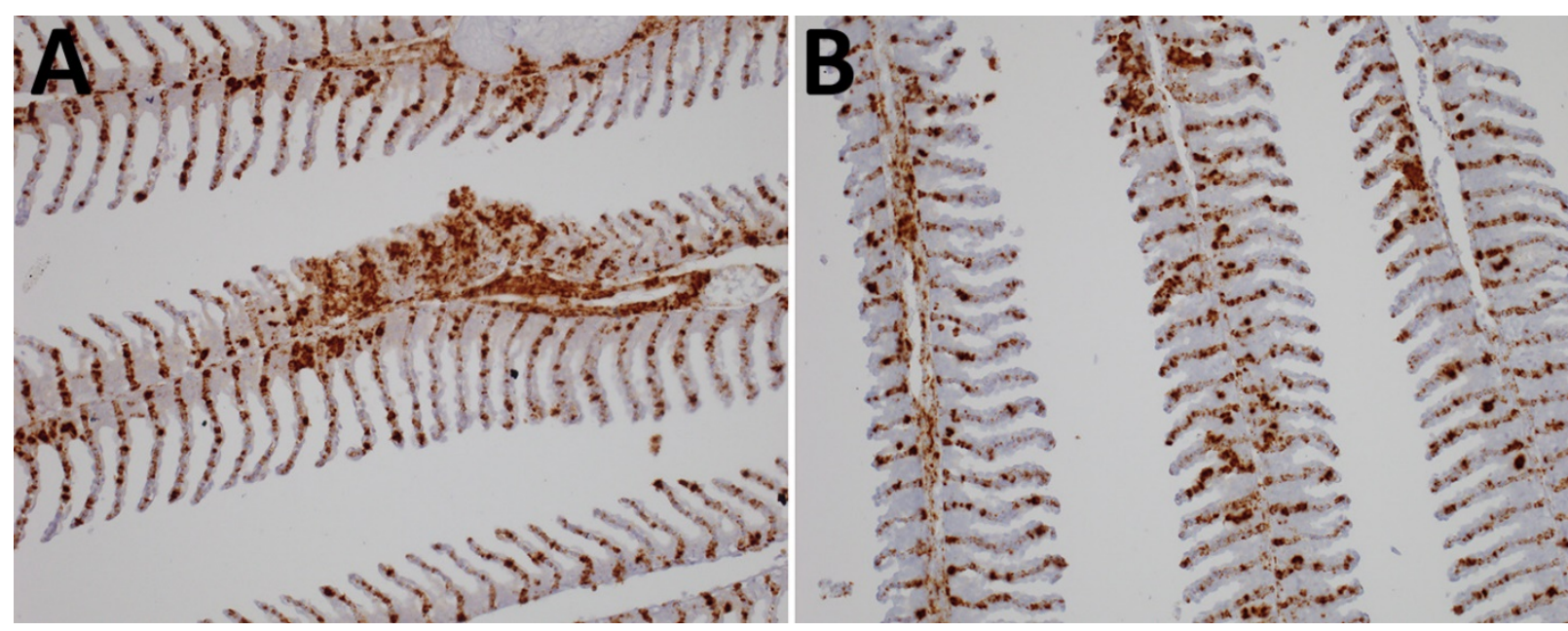

Appendix Figure 4. Detection of Bern perch virus (BRPV) genomic RNA in gills of two individual European perch (A, B) by in situ hybridization (brown labeling). 